\title{
CIRCULATORY DYNAMICS BEFORE AND AFTER EXERCISE IN SUBJECTS WITH AND WITHOUT STRUCTURAL HEART DISEASE DURING ANXIETY AND RELAXATION ${ }^{1}$
}

\author{
By IAN P. STEVENSON,2 CHARLES H. DUNCAN, AND HAROLD G. WOLFF
}

\author{
(From the New York Hospital and the Departments of Medicine and Psychiatry of Cornell \\ University Medical College, New York, N. Y.)
}

(Received for publication June 2, 1949)

Intolerance to exercise is a frequent complaint of patients with anxiety and their complaints of palpitations and dyspnea upon exertion have been related to objectively measured abnormalities in the circulation after exercise (1). Similar complaints and evidence of impaired exercise tolerance have been found in patients described under the heading of "neurocirculatory asthenia" or "effort syndrome" (2-4). Although the emphasis on symptoms in these two groups may be differently placed it is evident that the emotional and cardiovascular patterns are similar. Friedman observed that the impairment of exercise tolerance in patients with neurocirculatory asthenia was related to the presence of anxiety during the test rather than to any fundamental defect in the circulation (5). It has also been found that healthy subjects without complaints or evidence of cardiovascular disease will, under stressful conditions associated with disturbing emotional states such as anxiety and resentment, show impaired exercise tolerance as judged by excessive increases in heart rate, stroke volume or both during exercise (6). The present study was designed to investigate the relationships of these observations by exploring the occurrence and range of impaired exercise tolerance in a group of healthy subjects without complaints and patients with and without structural heart disease who had complaints of effort intolerance.

\section{METHODS}

The following indices were measured before and at intervals of two, three, five and ten minutes after a standard exercise: heart rate, stroke volume, and blood pressure. From these were calculated cardiac index and peripheral resistance. The heart rate and stroke volume

1 Supported in part by grants from the Commonwealth Fund and the Lester N. Hofheimer Foundation.

2 Present address: Department of Neuropsychiatry, Louisiana State University, School of Medicine, New Orleans 13, La. were measured on a low frequency, critically damped ballistocardiograph (7) and stroke volume was calculated by the formula of Nickerson (8). Blood pressure was taken by the auscultatory method. Mean blood pressure was calculated by adding $1 / 3$ of the pulse pressure to the diastolic pressure and peripheral resistance was derived by dividing the cardiac index into the mean pressure. The exercise performed was that devised by Master (9) which is standardized for the sex, age, and weight of the subject. In nearly every instance the tests were done in the basal condition; a few were performed at other times of the day but not less than two hours after a meal. The resting values were taken after a period of approximately 20 minutes rest which was spent partly lying on a comfortable bed and partly lying on the somewhat less comfortable ballistocardiograph table.

The emotional state of each subject at the time of the test was assessed from information derived in several ways. One index was the subject's own statements concerning his feeling state and awareness of emotional disturbance or tension. Secondly, inferences concerning his emotional state could be made from the conversation of the subject as it revealed his attitudes, pressure of talk, preoccupying or recurrent topics, current dreams, and associations thereto. Thirdly, the subject's behaviour, voice, movements and gestures provided information about his emotional state. Fourthly, information was obtained about the life situation of the subject and his reaction to it and to former life situations. In this connection the acquaintances of the subjects often gave information which corroborated or modified the opinions of the subject himself. Since the subjects were all either familiar laboratory workers or patients in the clinic of the hospital, a previous knowledge of their behavior and reactions aided the assessment of their emotional states which was composed from the assembled data.

Every effort was made to keep the circumstances of the test neutral and undisturbing to the patient. This was not always possible and in a few instances the test evoked tension or anxiety. In the remainder the emotional state was related to the general life situation of the subject and the test produced little or no modifications of this state. In a number of the patients exercise tolerance was tested before and after an interview in which were discussed pertinent life situations associated with the prevailing emotional state of the subject.

Inquiry was also made into the occurrence of symptoms before, during and after the exercise with particular attention to those commonly associated with effort in- 
tolerance such as palpitations, dyspnea, dizziness, and weakness.

\section{Subjects and Division of Material}

The subjects were drawn from several groups. Some were healthy members of the hospital staff without complaints. Others were patients of the hospital clinic with such complaints as palpitations, dyspnea, effort intolerance and others usually described as neurocirculatory asthenia. These two groups were without evidence of structural heart disease or diastolic hypertension. Other patients with evidence of structural heart disease or hypertension were also studied.

The subjects without structural heart disease or hypertension were divided into three groups according to the assessment of their emotional state by the methods described above.

The first group $(A)$ consisted of 18 subjects who were found by the methods of assessment described to be optimally relaxed at the time of the test. The second group $(B)$ consisted of 11 subjects who, for one reason or another, were unable to achieve complete relaxation. The initial statements of some of them implied that they were relaxed, but they were nevertheless found to have some preoccupation and slight emotional disturbance which prevented them from relaxing as fully as possible. It is to be stressed that at the time of the test tension was either minimal or altogether denied by these subjects and its presence inferred either from the anamnestic data, observation or subsequent revelations. Typical instances of the preoccupations noted were the following. One young physician felt that the technician took overly long in performing the test; two others were preoccupied with the heavy load of work which awaited them after the test; one received an unpleasant bill before leaving her house and another mulled over a disagreement which had occurred the night before. The significance of these preoccupations was shown by the attainment by many members of this group of complete relaxation and improved exercise tolerance at other times.

The third group $(C)$ consisted of 13 subjects with greater emotional disturbances amounting to marked, conscious anxiety. All but one of them were receiving treatment in the clinic of the New York Hospital for such anxiety and associated symptoms of palpitations, dyspnea and exercise intolerance.

\section{RESULTS}

\section{a) Cardiac Indices on Unselected Subjects}

Nickerson (using a similar ballistocardiograph) studied 50 healthy male medical students and 20 healthy female laboratory workers under basal conditions and with a rest period comparable to that used in this study. He found for males an average cardiac index of 3.4 and for females an average index of 2.7 (10). A similar unselected group of 16 healthy males and 12 healthy females studied in this laboratory gave average cardiac indices respectively of 3.3 and 2.7 , figures in close agreement with those of Nickerson. Both of these series were derived without consideration of the subjects' emotional states apart from the elimination of persons with overt anxiety.

\section{b) Comparison of Resting Values}

In group $A$ there were nine males and nine females. In group $B$ there were five males and six females. In group $C$ there were five males and eight females. Because it has been found by Nickerson (10) and by the present workers that there is a difference in cardiac output in males and females which is not absorbed by referring the output to the surface area (cardiac index), the averages of groups $B$ and $C$ have been adjusted for the proportions of males and females therein so that they may be compared with group $A$.

The average values obtained and the ranges observed are given in Table $I$ and outlined in diagrammatic form in Figure 1. A significant difference in resting cardiac indices between the three groups was found. Although there was considerable overlapping, group $B$ gave values intermediate between those of groups $A$ and $C$. Apart from the higher values for heart rate, stroke volume, and cardiac index found in group $C$, this group was set apart from the other two groups by the presence of a higher diastolic pressure and a peripheral resistance as great as that of group $B$. This indicates a failure of relaxation in peripheral vessels proportionate to the increase in cardiac output.

\section{c) Comparison of Responses to Standard Exercise}

As with resting values, "preoccupied" subjects and those with anxiety showed higher values for blood pressure, heart rate, and cardiac output after exercise than did the fully relaxed subjects. The higher cardiac outputs after exercise in the "preoccupied" compared to the relaxed subjects were largely attained by increases in stroke volume. In contrast, the higher cardiac outputs after exercise in subjects with anxiety compared to "preoccupied" subjects were largely attained by increases in heart rate. While the heart rate returned to within two beats per minute of the resting values by the end of two minutes in the relaxed subjects and by the end of three minutes in the "preoccupied" group, it had not quite returned to 
that level at the end of ten minutes in the subjects with anxiety. Although the resting values of heart rate and cardiac output of the "preoccupied" group were well within the normal range of such indices, impairment of exercise tolerance was nevertheless apparent in this group.

\section{d). Relation of Impaired Exercise Tolerance to Occurrence Symptoms}

In general there was a relation between impaired exercise tolerance and the occurrence of symptoms such as dyspnea and palpitations during and after

TABLE I

Exercise tolerance in different emotional states *

\begin{tabular}{|c|c|c|c|c|c|c|c|c|c|c|c|c|c|c|}
\hline \multirow{3}{*}{ Group } & & \multirow{3}{*}{ Age } & \multicolumn{6}{|c|}{ Resting } & \multicolumn{6}{|c|}{$2^{\prime}$ after exercise } \\
\hline & & & \multicolumn{2}{|c|}{ Blood press. } & \multirow{2}{*}{ Rate } & \multirow{2}{*}{$\begin{array}{l}\text { Stroke } \\
\text { volume }\end{array}$} & \multirow{2}{*}{$\mid \begin{array}{c}\text { Cardiac } \\
\text { index }\end{array}$} & \multirow{2}{*}{$\begin{array}{l}\text { Periph. } \\
\text { resist. }\end{array}$} & \multicolumn{2}{|c|}{ Blood press. } & \multirow{2}{*}{ Rate } & \multirow{2}{*}{$\begin{array}{l}\text { Stroke } \\
\text { volume }\end{array}$} & \multirow{2}{*}{$\begin{array}{c}\text { Cardiac } \\
\text { index }\end{array}$} & \multirow{2}{*}{$\begin{array}{l}\text { Periph. } \\
\text { resist. }\end{array}$} \\
\hline & & & Syst. & Diast. & & & & & Syst. & Diast. & & & & \\
\hline $\begin{array}{l}\text { A. Fully Relaxed } \\
\text { Nine Males } \\
\text { Nine Females }\end{array}$ & $\begin{array}{l}\text { Av. } \\
\text { Range High } \\
\text { Low }\end{array}$ & $\begin{array}{l}29 \\
46 \\
21\end{array}$ & $\begin{array}{r}103 \\
120 \\
94\end{array}$ & $\begin{array}{l}66 \\
76 \\
57\end{array}$ & $\begin{array}{l}64 \\
77 \\
50\end{array}$ & $\begin{array}{r}c c . \\
76 \\
113 \\
56\end{array}$ & $\begin{array}{c}\text { l./min./ } \\
\text { sq. } m . \\
2.6 \\
3.5 \\
1.7\end{array}$ & $\begin{array}{c}\underset{\text { l. } / \text { min. }}{\mathrm{H} g} \mathbf{|} \\
\text { sq. m. } \\
31 \\
48 \\
23\end{array}$ & $\begin{array}{r}112 \\
122 \\
95\end{array}$ & $\begin{array}{l}63 \\
75 \\
52\end{array}$ & $\begin{array}{l}65 \\
78 \\
51\end{array}$ & $\begin{array}{r}c c . \\
95 \\
136 \\
69\end{array}$ & \begin{tabular}{|c|} 
l. $/ \min . /$ \\
sq. $m$. \\
3.4 \\
4.9 \\
2.4
\end{tabular} & $\begin{array}{c}\operatorname{mm} . H g / \\
\text { l. } \mid \min . / \\
\text { sq. } m . \\
24 \\
33 \\
15\end{array}$ \\
\hline $\begin{array}{l}\text { B. "Preoccupied" or Poorly } \\
\text { Relaxed } \\
\text { Five Males } \\
\text { Six Females }\end{array}$ & $\begin{array}{l}\text { Av. } \\
\text { Range High } \\
\text { Low }\end{array}$ & $\begin{array}{l}29 \\
34 \\
20\end{array}$ & $\begin{array}{l}113 \\
137 \\
101\end{array}$ & $\begin{array}{l}68 \\
79 \\
59\end{array}$ & $\begin{array}{l}75 \\
89 \\
62\end{array}$ & $\begin{array}{r}90 \\
127 \\
59\end{array}$ & $\begin{array}{l}3.7 \\
4.7 \\
2.5\end{array}$ & $\begin{array}{l}24 \\
33 \\
14\end{array}$ & $\begin{array}{l}126 \\
157 \\
111\end{array}$ & $\begin{array}{l}67 \\
75 \\
61\end{array}$ & $\begin{array}{r}78 \\
109 \\
58\end{array}$ & $\begin{array}{r}122 \\
178 \\
91\end{array}$ & $\begin{array}{l}5.3 \\
7.2 \\
4.1\end{array}$ & $\begin{array}{l}17 \\
22 \\
13\end{array}$ \\
\hline $\begin{array}{l}\text { C. Anxiety } \\
\text { Five Males } \\
\text { Eight Females }\end{array}$ & $\begin{array}{l}\text { Av. } \\
\text { Range High }\end{array}$ & $\begin{array}{l}35 \\
48 \\
17\end{array}$ & $\begin{array}{l}128 \\
146 \\
105\end{array}$ & $\begin{array}{l}83 \\
93 \\
65\end{array}$ & $\begin{array}{r}90 \\
122 \\
74\end{array}$ & $\begin{array}{r}93 \\
126 \\
46\end{array}$ & $\begin{array}{l}4.8 \\
6.7 \\
2.6\end{array}$ & $\begin{array}{l}23 \\
39 \\
15\end{array}$ & $\begin{array}{l}140 \\
180 \\
113\end{array}$ & $\begin{array}{l}80 \\
92 \\
61\end{array}$ & $\begin{array}{r}102 \\
160 \\
79\end{array}$ & $\begin{array}{r}125 \\
158 \\
60\end{array}$ & $\begin{array}{r}7.5 \\
15.2 \\
4.1\end{array}$ & $\begin{array}{r}17 \\
30 \\
7\end{array}$ \\
\hline \multirow{3}{*}{ Group } & & \multirow{3}{*}{ Age } & \multicolumn{6}{|c|}{$3^{\prime}$ after exercise } & \multicolumn{6}{|c|}{$5^{\prime}$ after exercise } \\
\hline & & & \multicolumn{2}{|c|}{ Blood press. } & \multirow{2}{*}{ Rate } & \multirow{2}{*}{$\begin{array}{l}\text { Stroke } \\
\text { volume }\end{array}$} & \multirow{2}{*}{$\begin{array}{c}\text { Cardiac } \\
\text { index }\end{array}$} & \multirow{2}{*}{$\begin{array}{l}\text { Periph. } \\
\text { resist. }\end{array}$} & \multicolumn{2}{|c|}{ Blood press. } & \multirow{2}{*}{ Rate } & \multirow{2}{*}{$\begin{array}{l}\text { Stroke } \\
\text { volume }\end{array}$} & \multirow{2}{*}{$\begin{array}{c}\text { Cardiac } \\
\text { index }\end{array}$} & \multirow{2}{*}{$\begin{array}{l}\text { Periph. } \\
\text { resist. }\end{array}$} \\
\hline & & & Syst. & Diast. & & & & & Syst. & Diast. & & & & \\
\hline $\begin{array}{l}\text { A. Fully Relaxed } \\
\text { Nine Males } \\
\text { Nine Females }\end{array}$ & $\begin{array}{l}\text { Av. } \\
\text { Range High } \\
\text { Low }\end{array}$ & $\begin{array}{l}29 \\
46 \\
21\end{array}$ & $\begin{array}{r}109 \\
116 \\
91\end{array}$ & $\begin{array}{l}63 \\
72 \\
51\end{array}$ & $\begin{array}{l}64 \\
82 \\
53\end{array}$ & $\begin{array}{r}c c . \\
90 \\
139 \\
62\end{array}$ & \begin{tabular}{|c|} 
l./min./ \\
sq. $m$. \\
3.1 \\
4.9 \\
2.3
\end{tabular} & \begin{tabular}{|c|}
$\operatorname{mm} . \mathrm{Hg} /$ \\
l. $/ \min . /$ \\
sq. m. \\
26 \\
35 \\
15
\end{tabular} & $\begin{array}{r}105 \\
116 \\
93\end{array}$ & $\begin{array}{l}62 \\
73 \\
51\end{array}$ & $\begin{array}{l}63 \\
76 \\
50\end{array}$ & $\begin{array}{r}c c . \\
84 \\
129 \\
61\end{array}$ & \begin{tabular}{|c|} 
l. $/ \min . /$ \\
sq. $m$. \\
2.9 \\
4.0 \\
2.0
\end{tabular} & $\begin{array}{c}\underset{l}{\operatorname{mm} . \mathrm{Hg} /} \mathbf{m i n} . / \\
\text { sq. m. } \\
27 \\
40 \\
19\end{array}$ \\
\hline $\begin{array}{l}\text { B. "Preoccupied" or Poorly } \\
\text { Relaxed } \\
\text { Five Males } \\
\text { Six Females }\end{array}$ & $\begin{array}{l}\text { Av. } \\
\text { Range High } \\
\text { Low }\end{array}$ & $\begin{array}{l}29 \\
34 \\
20\end{array}$ & $\begin{array}{l}120 \\
152 \\
109\end{array}$ & $\begin{array}{l}67 \\
78 \\
58\end{array}$ & $\begin{array}{r}77 \\
104 \\
57\end{array}$ & $\begin{array}{r}116 \\
176 \\
77\end{array}$ & $\begin{array}{l}4.8 \\
7.1 \\
3.1\end{array}$ & $\begin{array}{l}18 \\
29 \\
13\end{array}$ & $\begin{array}{l}118 \\
149 \\
103\end{array}$ & $\begin{array}{l}66 \\
78 \\
58\end{array}$ & $\begin{array}{l}74 \\
91 \\
57\end{array}$ & $\begin{array}{r}102 \\
147 \\
71\end{array}$ & $\begin{array}{r}-4.2 \\
5.3 \\
2.9\end{array}$ & $\begin{array}{l}21 \\
30 \\
17\end{array}$ \\
\hline $\begin{array}{l}\text { C. Anxiety } \\
\text { Five Males } \\
\text { Eight Females }\end{array}$ & $\begin{array}{l}\text { Av. } \\
\text { Range High } \\
\text { Low }\end{array}$ & $\begin{array}{l}35 \\
48 \\
17\end{array}$ & $\begin{array}{l}134 \\
175 \\
108\end{array}$ & $\begin{array}{l}80 \\
91 \\
61\end{array}$ & $\begin{array}{r}100 \\
150 \\
84\end{array}$ & $\begin{array}{r}110 \\
142 \\
58\end{array}$ & $\begin{array}{r}6.4 \\
11.9 \\
3.6\end{array}$ & $\begin{array}{r}18 \\
33 \\
9\end{array}$ & $\begin{array}{l}131 \\
167 \\
102\end{array}$ & $\begin{array}{l}80 \\
93 \\
62\end{array}$ & $\begin{array}{r}95 \\
135 \\
77\end{array}$ & $\begin{array}{r}102 \\
141 \\
50\end{array}$ & $\begin{array}{r}5.6 \\
10.1 \\
2.7\end{array}$ & $\begin{array}{l}21 \\
36 \\
11\end{array}$ \\
\hline \multirow{3}{*}{ Group } & & \multirow{3}{*}{ Age } & \multicolumn{6}{|c|}{$10^{\prime}$ after exercise } & & & & & & \\
\hline & & & \multicolumn{2}{|c|}{ Blood press. } & \multirow{2}{*}{ Rate } & & & & & & & & & \\
\hline & & & Syst. & Diast. & & volume & index & resist & & • & & & & \\
\hline $\begin{array}{l}\text { A. Fully Relaxed } \\
\text { Nine Males } \\
\text { Nine Females }\end{array}$ & $\begin{array}{l}\text { Av. } \\
\text { Range High } \\
\text { Low }\end{array}$ & $\begin{array}{l}29 \\
46 \\
21\end{array}$ & $\begin{array}{r}103 \\
111 \\
93\end{array}$ & $\begin{array}{l}65 \\
72 \\
57\end{array}$ & $\begin{array}{l}64 \\
73 \\
50\end{array}$ & $\begin{array}{r}c c . \\
78 \\
116 \\
57\end{array}$ & \begin{tabular}{|c|} 
l. $/$ min. \\
sq. $m$. \\
2.7 \\
3.6 \\
2.0
\end{tabular} & $\begin{array}{c}\text { mm. } H g / \\
\text { l./min./ } \\
\text { sq. m. } \\
29 \\
39 \\
20\end{array}$ & & & & & & \\
\hline $\begin{array}{l}\text { B. "Preoccupied" or Poorly } \\
\text { Relaxed } \\
\text { Five Males } \\
\text { Six Females }\end{array}$ & $\begin{array}{l}\text { Av. } \\
\text { Range High } \\
\text { Low }\end{array}$ & $\begin{array}{l}29 \\
34 \\
20\end{array}$ & $\begin{array}{l}110 \\
133 \\
101\end{array}$ & $\begin{array}{l}69 \\
79 \\
59\end{array}$ & $\begin{array}{l}74 \\
90 \\
61\end{array}$ & $\begin{array}{r}88 \\
134 \\
62\end{array}$ & $\begin{array}{l}3.6 \\
4.4 \\
2.8\end{array}$ & $\begin{array}{l}24 \\
32 \\
19\end{array}$ & & & . & & & \\
\hline $\begin{array}{l}\text { C. Anxiety } \\
\text { Five Males } \\
\text { Eight Females }\end{array}$ & $\begin{array}{l}\text { Av. } \\
\text { Range High } \\
\text { Low }\end{array}$ & $\begin{array}{l}35 \\
48 \\
17\end{array}$ & $\begin{array}{l}129 \\
156 \\
105\end{array}$ & $\begin{array}{l}81 \\
94 \\
63\end{array}$ & $\begin{array}{r}93 \\
127 \\
76\end{array}$ & $\begin{array}{r}91 \\
124 \\
53\end{array}$ & $\begin{array}{l}4.9 \\
8.7 \\
2.6\end{array}$ & $\begin{array}{l}23 \\
38 \\
13\end{array}$ & & & & & & \\
\hline
\end{tabular}

* The mean figures before and after standard exercise are given for three groups, into which the subjects were placed according to their emotional state. 
the exercise. Subjects with anxiety usually had such complaints, the relaxed subjects rarely. Some of the "preoccupied" subjects, however, were unaware of impaired exercise tolerance in themselves just as they often ignored or considered unimportant their disturbed emotional state. Others noted that the exercise seemed easier on days of relaxation than when they were "preoccupied."

\section{e) Variations in Exercise Tolerance in Individual Subjects During Different Emotional States}

Many of the subjects of this study with and without structural heart disease were studied during different life situations and emotional states over a period up to 18 months. In each of 25 subjects a close correlation was found between the emotional state and exercise tolerance. During periods of remission from anxiety it was frequently found that the resting values of cardiac output and heart rate would be normal while the exercise tolerance continued to be impaired. With further improvement the exercise tolerance approached or attained normality although it was unusual for subjects with anxiety to improve further than the performance of the "preoccupied" subjects. As in earlier studies (6) the pulse rate was found to be an unreliable index of exercise tolerance since in the preoccupied group and in some subjects with

TABLE II

Circulatory dynamics before and after exercise during anxiety and relaxation *

\begin{tabular}{|c|c|c|c|c|c|c|c|c|c|c|c|c|c|c|c|c|c|}
\hline \multirow{3}{*}{ Patient } & \multirow{3}{*}{ Age } & \multirow{3}{*}{ Sex } & \multirow{3}{*}{ Diagnosis } & \multirow{3}{*}{$\begin{array}{l}\text { Emo- } \\
\text { tional } \\
\text { state }\end{array}$} & \multirow{3}{*}{ Date } & \multicolumn{6}{|c|}{ Resting } & \multicolumn{6}{|c|}{ After Exercise } \\
\hline & & & & & & \multicolumn{2}{|c|}{ Blood press. } & \multirow{2}{*}{$\begin{array}{c}\text { Heart } \\
\text { rate }\end{array}$} & \multirow{2}{*}{$\begin{array}{l}\text { Stroke } \\
\text { volume }\end{array}$} & \multirow{2}{*}{$\begin{array}{c}\text { Cardiac } \\
\text { index }\end{array}$} & \multirow{2}{*}{$\begin{array}{c}\text { Periph. } \\
\text { resist. }\end{array}$} & \multicolumn{2}{|c|}{ Blood press. } & \multirow{2}{*}{$\begin{array}{c}\text { Heart } \\
\text { rate }\end{array}$} & \multirow{2}{*}{$\begin{array}{l}\text { Stroke } \\
\text { volume }\end{array}$} & \multirow{2}{*}{$\begin{array}{c}\text { Cardiac } \\
\text { index }\end{array}$} & \multirow{2}{*}{$\begin{array}{l}\text { Periph. } \\
\text { resist. }\end{array}$} \\
\hline & & & & & & Syst. & Diast. & & & & & Syst. & Diast. & & & & \\
\hline 1. B.A. & 17 & F & Anxiety & $\begin{array}{l}\text { Relaxed } \\
\text { Anxious }\end{array}$ & $\begin{array}{l}3 / 5 / 49 \\
11 / 20 / 48\end{array}$ & $\begin{array}{c}\mathrm{mm} . \\
\mathrm{Hg} \\
116 \\
146\end{array}$ & $\begin{array}{c}m m . \\
H g \\
67 \\
90\end{array}$ & $\begin{array}{r}97 \\
122\end{array}$ & $\begin{array}{r}c c . \\
100 \\
78\end{array}$ & $\mid \begin{array}{c}\text { l.min. } \\
\text { sq. } m . \\
5.8 \\
5.7\end{array}$ & \begin{tabular}{|c|} 
mm. $\mathrm{Hg} /$ \\
l./min. \\
sq. m. \\
14 \\
18
\end{tabular} & $\begin{array}{c}\underset{H g}{m m} \\
144 \\
180\end{array}$ & $\begin{array}{c}\underset{H g}{m m} . \\
65 \\
75\end{array}$ & $\begin{array}{l}110 \\
160\end{array}$ & $\begin{array}{l}c c . \\
171 \\
158\end{array}$ & $\begin{array}{c}l . / \min . / \\
\text { sq. } m . \\
11.3 \\
15.2\end{array}$ & \begin{tabular}{|c}
$\operatorname{mm.Hg} \mid$ \\
l./min./ \\
sq. m. \\
8 \\
7
\end{tabular} \\
\hline 2. F.S. & 38 & F & Anxiety & $\begin{array}{l}\text { Relaxed } \\
\text { Anxious }\end{array}$ & $\begin{array}{c}4 / 10 / 48 \\
1 \mathrm{hr} . \\
\text { earlier }\end{array}$ & $\begin{array}{l}130 \\
138\end{array}$ & $\begin{array}{l}85 \\
78\end{array}$ & $\begin{array}{r}95 \\
108\end{array}$ & $\begin{array}{r}88 \\
107\end{array}$ & $\begin{array}{l}5.1 \\
7.0\end{array}$ & $\begin{array}{l}20 \\
14\end{array}$ & $\begin{array}{l}125 \\
136\end{array}$ & $\begin{array}{l}75 \\
78\end{array}$ & $\begin{array}{l}104 \\
118\end{array}$ & $\begin{array}{l}125 \\
121\end{array}$ & $\begin{array}{l}7.9 \\
8.7\end{array}$ & $\begin{array}{l}13 \\
11\end{array}$ \\
\hline 3. S.F. & 48 & F & Anxiety & $\begin{array}{l}\text { Relaxed } \\
\text { Anxious }\end{array}$ & $\begin{array}{l}3 / 12 / 49 \\
9 / 21 / 48\end{array}$ & $\begin{array}{l}159 \\
139\end{array}$ & $\begin{array}{l}91 \\
86\end{array}$ & $\begin{array}{l}88 \\
89\end{array}$ & $\begin{array}{l}72 \\
81\end{array}$ & $\begin{array}{l}4.5 \\
5.0\end{array}$ & $\begin{array}{l}25 \\
21\end{array}$ & $\begin{array}{l}168 \\
155\end{array}$ & $\begin{array}{l}85 \\
85\end{array}$ & $\begin{array}{r}90 \\
100\end{array}$ & $\begin{array}{l}115 \\
111\end{array}$ & $\begin{array}{l}7.4 \\
7.7\end{array}$ & $\begin{array}{l}15 \\
14\end{array}$ \\
\hline \multirow[t]{2}{*}{ 4. M.V. } & \multirow[t]{2}{*}{34} & \multirow[t]{2}{*}{ F } & \multirow{2}{*}{$\begin{array}{l}\text { Mitral } \\
\text { stenosis } \\
\text { Aortic } \\
\text { insuff. }\end{array}$} & Relaxed & $3 / 30 / 49$ & 111 & 74 & 67 & 62 & 2.2 & 40 & 135 & 78 & 91 & 116 & 5.5 & 18 \\
\hline & & & & Anxious & $5 / 14 / 48$ & 120 & 70 & 89 & 102 & 4.8 & 18 & 143 & 73 & 106 & 151 & 8.5 & 11 \\
\hline \multirow[t]{2}{*}{ 5. I.D. } & \multirow[t]{2}{*}{32} & \multirow[t]{2}{*}{ F } & \multirow{2}{*}{$\begin{array}{l}\text { Mitral } \\
\text { stenosis }\end{array}$} & Relaxed & $4 / 27 / 49$ & 100 & 66 & 75 & 66 & 3.4 & 23 & 111 & 65 & 73 & 123 & 6.1 & 13 \\
\hline & & & & Anxious & $11 / 3 / 48$ & 118 & 70 & 100 & 68 & 4.7 & 18 & 121 & 75 & 106 & 119 & 8.7 & 10 \\
\hline 6. J.C. & 45 & $\mathbf{M}$ & Anxiety & $\begin{array}{l}\text { Relaxed } \\
\text { Anxious }\end{array}$ & $\begin{array}{l}11 / 6 / 48 \\
7 / 16 / 48\end{array}$ & $\begin{array}{l}120 \\
127\end{array}$ & $\begin{array}{l}76 \\
84\end{array}$ & $\begin{array}{l}70 \\
90\end{array}$ & $\begin{array}{r}84 \\
100\end{array}$ & $\begin{array}{l}3.0 \\
4.6\end{array}$ & $\begin{array}{l}30 \\
22\end{array}$ & $\begin{array}{l}138 \\
139\end{array}$ & $\begin{array}{l}84 \\
86\end{array}$ & $\begin{array}{l}81 \\
94\end{array}$ & $\begin{array}{r}92 \\
134\end{array}$ & $\begin{array}{l}3.8 \\
6.4\end{array}$ & $\begin{array}{l}27 \\
16\end{array}$ \\
\hline 7. L.H. & 62 & $\mathbf{F}$ & $\begin{array}{l}\text { Art'scl. } \\
\text { Ht. dis. }\end{array}$ & $\begin{array}{l}\text { Relaxed } \\
\text { Angry }\end{array}$ & $\begin{array}{c}1 / 14 / 49 \\
1 \text { hr. } \\
3 \text { later } \\
\text { later }\end{array}$ & $\begin{array}{l}204 \\
250\end{array}$ & $\begin{array}{r}94 \\
112\end{array}$ & $\begin{array}{l}60 \\
68\end{array}$ & $\begin{array}{r}105 \\
96\end{array}$ & $\begin{array}{l}3.6 \\
3.7\end{array}$ & $\begin{array}{l}36 \\
43\end{array}$ & $\begin{array}{l}238 \\
249\end{array}$ & $\begin{array}{l}100 \\
107\end{array}$ & $\begin{array}{l}70 \\
74\end{array}$ & $\begin{array}{l}102 \\
112\end{array}$ & $\begin{array}{l}4.1 \\
4.7\end{array}$ & $\begin{array}{l}36 \\
33\end{array}$ \\
\hline 8. V.C. & 32 & $\mathbf{M}$ & Anxiety & $\begin{array}{l}\text { Relaxed } \\
\text { Anxious }\end{array}$ & $\begin{array}{l}1 / 13 / 49 \\
11 / 3 / 48\end{array}$ & $\begin{array}{l}124 \\
132\end{array}$ & $\begin{array}{l}66 \\
80\end{array}$ & $\begin{array}{l}82 \\
90\end{array}$ & $\begin{array}{l}140 \\
110\end{array}$ & $\begin{array}{l}5.9 \\
5.1\end{array}$ & $\begin{array}{l}14 \\
19\end{array}$ & $\begin{array}{l}130 \\
146\end{array}$ & $\begin{array}{l}64 \\
75\end{array}$ & $\begin{array}{r}92 \\
114\end{array}$ & $\begin{array}{l}161 \\
136\end{array}$ & $\begin{array}{l}7.6 \\
8.0\end{array}$ & $\begin{array}{l}11 \\
12\end{array}$ \\
\hline 9. J.B. & 29 & $\mathbf{M}$ & Anxiety & $\begin{array}{l}\text { Relaxed } \\
\text { Anxious }\end{array}$ & $\begin{array}{l}1 / 12 / 49 \\
11 / 18 / 48\end{array}$ & $\begin{array}{l}127 \\
129\end{array}$ & $\begin{array}{l}91 \\
89\end{array}$ & $\begin{array}{l}73 \\
77\end{array}$ & $\begin{array}{r}88 \\
126\end{array}$ & $\begin{array}{l}3.1 \\
4.6\end{array}$ & $\begin{array}{l}33 \\
22\end{array}$ & $\begin{array}{l}138 \\
144\end{array}$ & $\begin{array}{l}93 \\
92\end{array}$ & $\begin{array}{l}78 \\
85\end{array}$ & $\begin{array}{l}123 \\
155\end{array}$ & $\begin{array}{l}4.6 \\
6.3\end{array}$ & $\begin{array}{l}23 \\
17\end{array}$ \\
\hline 10. M.L. & 32 & F & Anxiety & $\begin{array}{l}\text { Relaxed } \\
\text { Anxious }\end{array}$ & $\begin{array}{c}2 / 27 / 48 \\
1 \mathrm{hr} . \\
\text { earlier }\end{array}$ & $\begin{array}{l}120 \\
124\end{array}$ & $\begin{array}{l}74 \\
84\end{array}$ & $\begin{array}{r}83 \\
102\end{array}$ & $\begin{array}{l}89 \\
91\end{array}$ & $\begin{array}{l}4.8 \\
6.1\end{array}$ & $\begin{array}{l}19 \\
16\end{array}$ & $\begin{array}{l}127 \\
120\end{array}$ & $\begin{array}{l}71 \\
80\end{array}$ & $\begin{array}{r}87 \\
112\end{array}$ & $\begin{array}{l}127 \\
119\end{array}$ & $\begin{array}{l}7.3 \\
8.8\end{array}$ & $\begin{array}{l}12 \\
11\end{array}$ \\
\hline 11. B.W. & 45 & F & $\begin{array}{l}\text { Mitral } \\
\text { stenosis }\end{array}$ & $\begin{array}{l}\text { Relaxed } \\
\text { Anxious }\end{array}$ & $\begin{array}{l}5 / 11 / 49 \\
9 / 28 / 48\end{array}$ & $\begin{array}{l}115 \\
121\end{array}$ & $\begin{array}{l}64 \\
66\end{array}$ & $\begin{array}{l}56 \\
60\end{array}$ & $\begin{array}{l}68 \\
116\end{array}$ & $\begin{array}{l}2.7 \\
4.9\end{array}$ & $\begin{array}{l}30 \\
17\end{array}$ & $\begin{array}{l}136 \\
135\end{array}$ & $\begin{array}{l}63 \\
70\end{array}$ & $\begin{array}{l}76 \\
83\end{array}$ & $\begin{array}{r}88 \\
145\end{array}$ & $\begin{array}{l}4.6 \\
8.6\end{array}$ & $\begin{array}{l}19 \\
11\end{array}$ \\
\hline 12. L.S. & 22 & F & \begin{tabular}{|l|} 
Patent \\
ductus \\
arteriosus
\end{tabular} & $\begin{array}{l}\text { Relaxed } \\
\text { Anxious }\end{array}$ & $\begin{array}{l}12 / 20 / 48 \\
2 / 26 / 48\end{array}$ & $\begin{array}{l}100 \\
128\end{array}$ & $\begin{array}{l}68 \\
78\end{array}$ & $\begin{array}{l}88 \\
99\end{array}$ & $\begin{array}{r}63 \\
124\end{array}$ & $\begin{array}{l}4.0 \\
9.9\end{array}$ & $\begin{array}{l}20 \\
10\end{array}$ & $\begin{array}{l}108 \\
128\end{array}$ & $\begin{array}{l}64 \\
65\end{array}$ & $\begin{array}{l}122 \\
132\end{array}$ & $\begin{array}{l}117 \\
196\end{array}$ & $\begin{array}{l}10.3 \\
18.5\end{array}$ & $\begin{array}{l}8 \\
5\end{array}$ \\
\hline
\end{tabular}

* The term "relaxed" merely contrasts the state with the other one of that subject and does not imply complete relaxation.

† The figures obtained after exercise were obtained at $2^{\prime}$ after the standard exercise in every case except Case 10 for whom (for technical reasons) the $3^{\prime}$ determinations are given.

¥ Case 8 (V. C.) showed a higher cardiac output at rest on his relaxéd day than when anxious. On the former day he rested only $10^{\prime}$ before the test, having rested $20^{\prime}$ on the day of greater anxiety. 


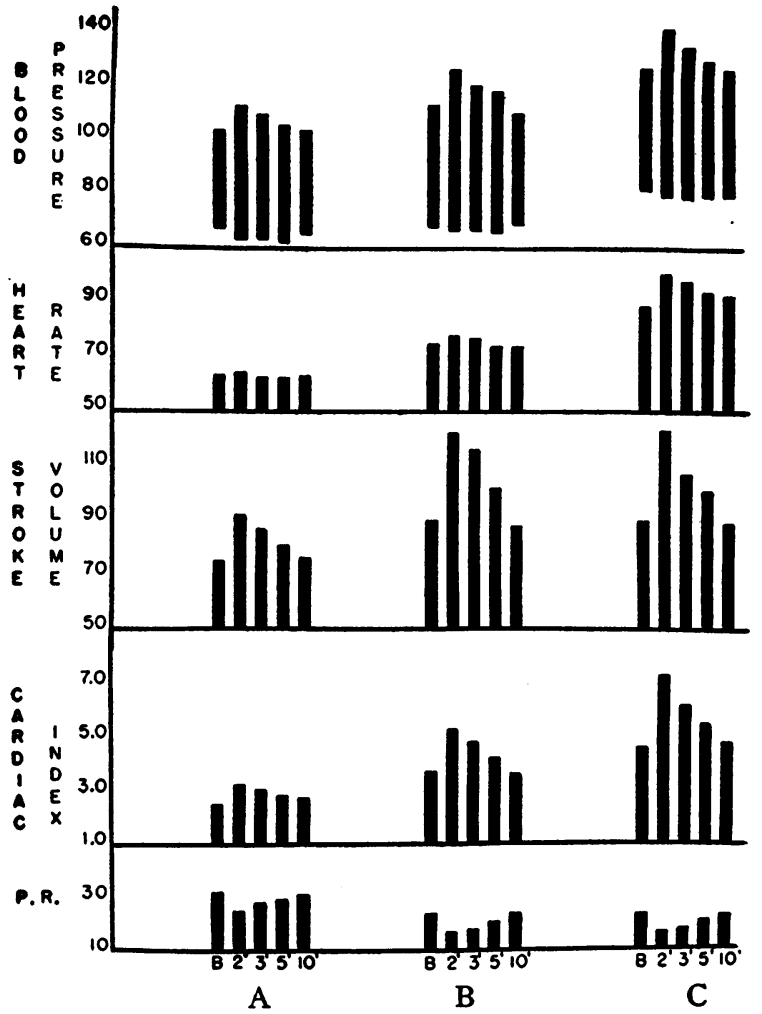

Fig. 1. Circulatory Dynamics in Different Emotional States

Indices of circulatory function are given for three groups before and after (two minutes, three minutes, five minutes, and ten minutes) exercise. Values given are the means for the groups (given in Table I). Group $A$ consisted of subjects optimally relaxed. Group $B$ of subjects poorly relaxed with preoccupations and mild psychic tension. Group $C$ of subjects with marked, conscious anxiety. No patients with structural heart disease were included in these groups. P.R.-Peripheral resistance.

anxiety, the heart rate returned to the resting values within two or three minutes while the stroke volume and cardiac index remained elevated for considerably longer.

Studies of individual patients brought out further the relative importance of increases in rate and stroke volume in the performance of exercise during anxiety and relaxation. The cardiac index was invariably less after exercise during relative relaxation, than during anxiety but the stroke volume was rarely much decreased and sometimes actually increased over that during anxiety. In many subjects decrease in cardiac index was achieved largely or entirely by a decrease in rate during relaxation compared to that during anxiety. These differences are evident in Table II which gives the figures obtained in 12 subjects before and two minutes after the standard exercise during periods of emotional disturbance and relaxation. A typical example of the changes found is illustrated in Figure 2 (Case 1).

\section{f) Rapid Variations in Exercise Tolerance}

In five instances it was found possible to improve exercise tolerance abruptly (i.e., in less than an hour) by strong reassurance and suggestions of relaxation or by listening sympathetically to a free expression by the subject of troublesome personal conflicts. Figure 3 illustrates changes in exercise tolerance occurring after such an interview in Case 10 of Table II. The patient was initially tense and anxious. During the interview she ventilated resentment felt against her husband, conflict with

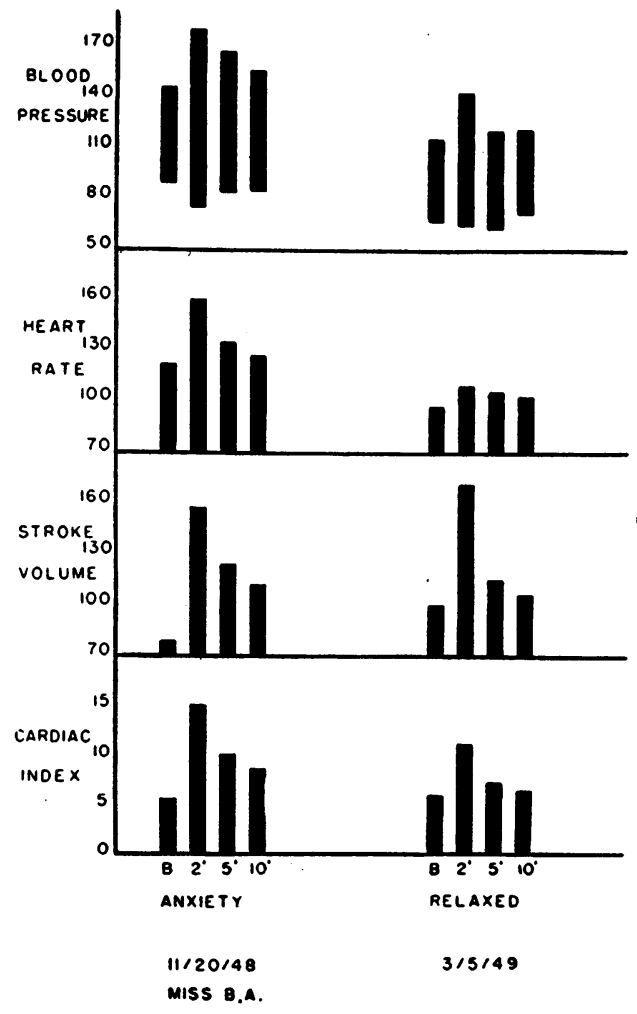

Fig. 2. Variations in Exercise Tolerance (CAse 1 of Table II)

Circulatory indices before and after standard exercise during anxiety and relative relaxation. During relaxation heart rate was decreased and stroke volume actually increased before and two minutes after the exercise. 


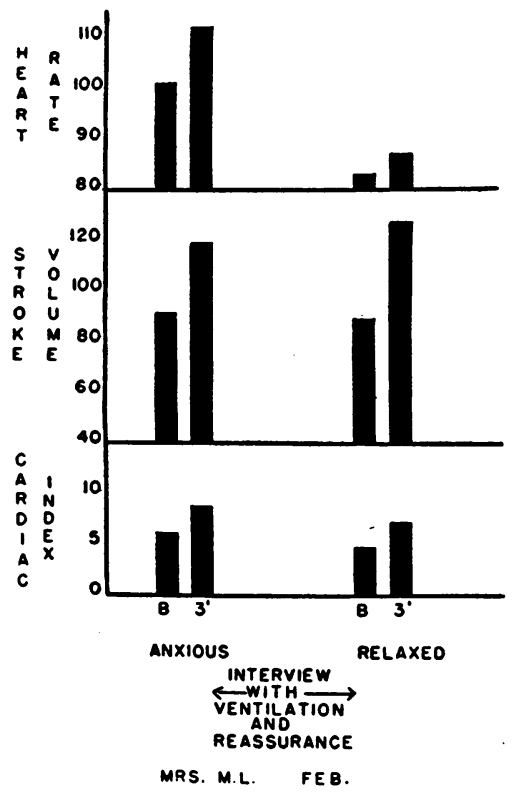

Fig. 3. Rapid Variation in Exercise Tolerance (CASE 10 OF TABLE II)

After the interview with ventilation of resentment and reassurance the patient was relaxed and circulatory efficiency improved as indicated by performance of identical exercise with slower heart rate and greater stroke volume.

whom had been the stressful life situation associated with the development of her palpitations. At the end of the interview exercise was performed with less increase in cardiac output chiefly because of a reduced heart rate. Stroke volume was actually higher after the exercise during relaxation than during anxiety.

It was shown that such results were not due to the gradual diminution in metabolism which might occur in a patient lying on the table for some time by the fact that in three patients in whom it was impossible to induce adequate relaxation, exercise tolerance on the second trial was no greater than it had been earlier. Furthermore in two patients thus studied there was initial relaxation, and impairment in exercise tolerance occurred after interviews in which the patients became respectively anxious and resentful. Case 7 of Table II is an instance of this phenomenon.

\section{g) Variations in Exercise Tolerance in Patients with Structural Heart Disease}

Ten patients with various forms of structural heart disease were studied and the results were similar to those obtained in patients without structural heart disease. In these instances the patient's complaints of palpitations, dyspnea and weakness on exertion could often be reasonably ascribed to the extensive structural heart disease found. Such symptoms were found to vary markedly with the emotional state of the patient and frequently disappeared altogether with the abolition of anxiety although the structural defects of the heart remained unchanged. Two examples of these changes may be briefly cited.

A 22 year old girl (Case 12 of Table II) came to the hospital with complaints of weakness, dyspnea, palpitations, and dizziness. She had always been warned to "take it easy" since the discovery of a heart murmur in childhood. Later as a result of an unsatisfactory love affair she became depressed and developed palpitations which in turn focussed anxiety on her heart. Nevertheless

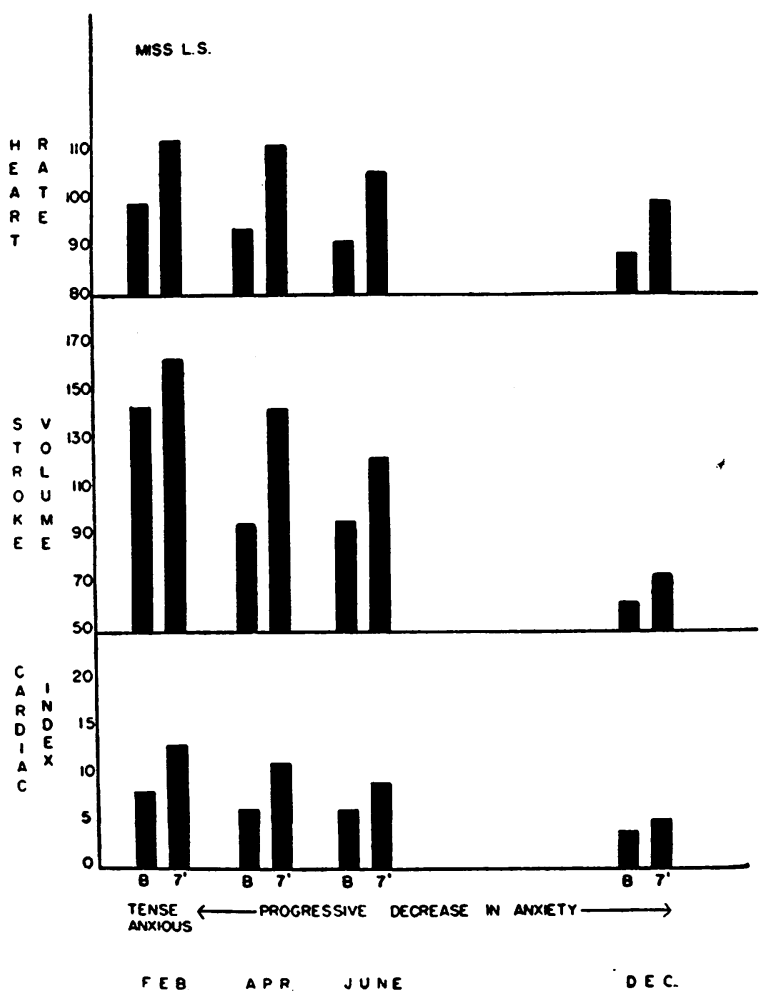

Fig. 4. Variations in Exercise Tolerance in Congenital Heart Disease (Patent Ductus ArteRIOSUS, CASE 12 OF TABLE II)

Circulatory indices are shown before and after standard exercise on four different occasions of a ten month period. Some intolerance to exercise is still present even during rather complete relaxation. 
her complaints of effort intolerance could have been (and were by some observers) attributed to patent ductus arteriosus which was demonstrated by angiocardiography. The patient was followed and studied over a ten month period. She gradually became more relaxed and concomitantly her symptoms disappeared and exercise tolerance improved (Figure 4). At the last test she had only mild dyspnea on climbing stairs and her other symptoms had disappeared altogether. She was leading a completely normal life. Her exercise tolerance was still slightly impaired presumably due to the congenital heart disease, but that portion of her impairment of cardiovascular function related to anxiety had been dissected off during her gradual relaxation.

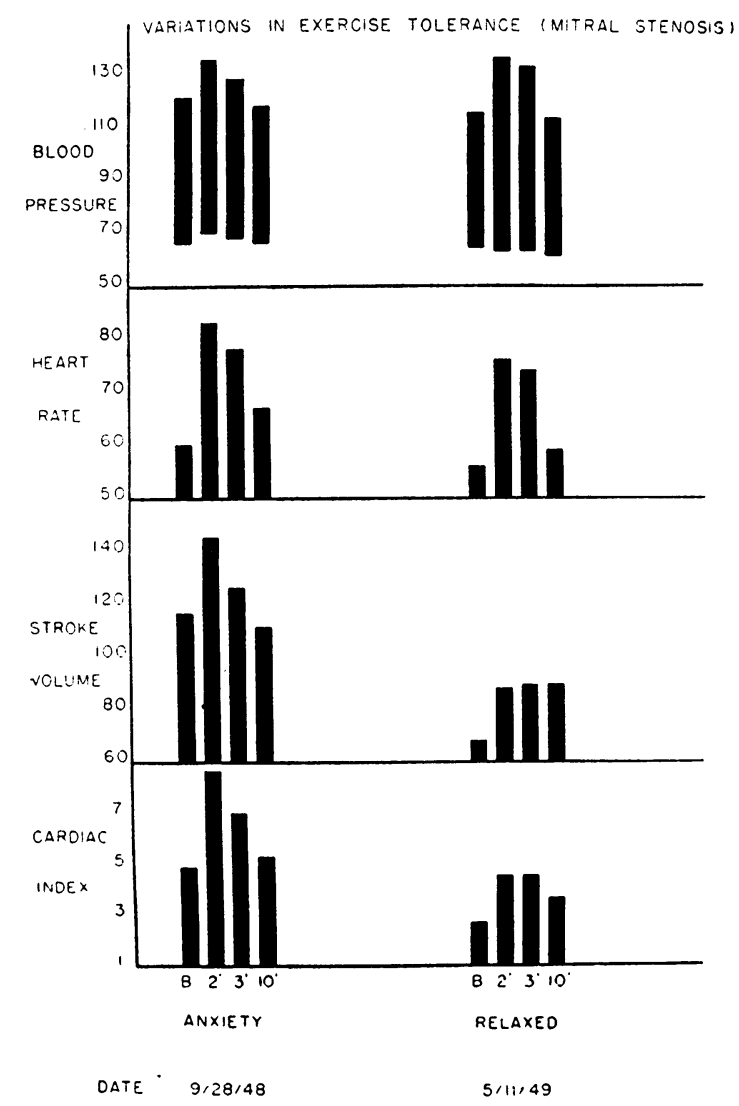

Fig. 5. Variations in Exercise Tolerance in Rheumatic Heart Disease (Mitral Stenosis, Case 11 of TABLE II)

Circulatory measurements before and after a standard exercise on two occasions are given. On the first day the patient had considerable anxiety. On the second day she was relaxed.
A 45 year old housewife (Case 11 of Table II) came to the hospital with complaints of palpitations both at rest and upon exertion, which she had had for four years. She was found to have an enlarged heart with mitral stenosis and insufficiency. The palpitations were attributable partly to extrasystoles and partly to increased heart rate and stroke volume. For almost 20 years prior to the onset of her symptoms the patient had wavered in her attachment to two men. One she had loved, but feeling unsure of him, she had married the other who appeared to be stronger but proved to be also unkind. As her husband increasingly maltreated her she finally resolved to divorce him. In the setting of this decision she became aware of marked tension and anxiety and noted the onset of her symptoms. These continued as her conflict was prolonged by her inability to detach herself from her husband (following the divorce) and marry the second man. Anxiety was joined by resentment when she blamed both men for her unhappiness. An exercise tolerance test at the height of her anxiety is shown in Figure 5. Following this the patient came to the clinic over a seven month period during which she ventilated and discussed her conflicts and the origins of her emotional disturbance. During this period she gradually became more relaxed and all her symptoms greatly improved. A second test of exercise tolerance is also shown in Figure 5. The resting heart rates on the two occasions are almost similar, but the resting stroke volumes and the response to the exercise show considerable differences.

A detailed study of the life situations and emotional states associated with the observed changes in exercise tolerance is published elsewhere (11).

\section{DISCUSSION}

Friedman has suggested that exertional tachycardia in anxiety is attributable to the associated tachypnea (5). In subjects of the present study however, tachycardia on exertion was often encountered in the absence of unusual tachypnea.

In view of the gradation in exercise tolerance from completely relaxed individuals through somewhat less relaxed subjects to those with overt anxiety, it seems likely that the impaired exercise tolerance in these subjects is related to a general mobilization for action involving the cardiovascu- 
lar system in response to a threat in the life situation (12). In our culture physical action appropriate to the stimulus rarely takes place, but cardiovascular mobilization seems to be invoked in response even to a symbolic stimulus when no action is intended. In the early stages of such mobilization, increased cardiac output is usually achieved largely by increases in stroke volume. In the more extreme degrees, increases in heart rate contribute more to the augmentation of cardiac output.

In some patients who were tense before the exercise, the latter procedure was diverting and relaxing to them. In such instances the cardiac output fell after the exercise. Hickam, Cargill, and Golden (13) observed such falls in cardiac output after exercise in some of their subjects in whom anxiety was artificially induced and in whom the ensuing exercise might have been diverting and relaxing. When anxiety persists during exercise there is an inappropriate and exaggerated response of cardiac function. When the stressful stimulus is removed or the subject's changed attitudes make it appear less stressful this exaggerated response disappears.

The findings of this study may be relevant to the relationship between tachycardia and the subsequent development of hypertension and structural heart disease to which patients with tachycardia are more than usually susceptible (14). The patients in this series with anxiety showed diastolic blood pressures appreciably higher than those of normal subjects. Since there is in general a decreased peripheral resistance during anxiety $(13,15)$, the presence of a peripheral resistance in these patients as great as that of the poorly relaxed subjects with considerably lower cardiac outputs indicates either that the limit of dilatation in the peripheral vessels had been reached or that part of this vasculature had constricted. Smith (16) and Wolf et al. (17) have shown that during anxiety there is a diminished renal blood flow which may occur in the presence of an increased cardiac output (17) and be sustained for some time after the disappearance of overt anxiety. These various studies suggest that during a period of stress a constriction of the splanchnic bed occurs which, although perhaps originally compensatory for the decrease in peripheral resistance elsewhere, may persist and, in- creasing with each new episode of anxiety, end in the establishment of a sustained hypertension.

Similarly the later occurrence of structural heart disease in patients with tachycardia may be related to the chronic overactivity of the heart shown by patients with anxiety. Although no measurements of cardiac functions could be made during or immediately after the exercise by this method, it is apparent from Table I and Figure 1 that subjects with anxiety had much higher cardiac outputs at rest than did relaxed subjects two minutes after moderately strenuous exercise. Further, the excess work in the patients with anxiety was associated with considerable tachycardia. Harrison has reviewed the ample evidence of the penalties paid by the heart which is operating at excessive rates (18). The tachycardia of the patients with anxiety here was perhaps not great, their heart rates averaging only 102 at two minutes after the exercise. But the average heart rates of completely.relaxed subjects after the same exercise was 65,37 beats per minute less.

In addition the duration as well as the degree of the cardiac hyperactivity at rest and after exercise must be considered. The patients with anxiety were observed to show repeated tachycardia during clinic visits and these represented stress which was trivial compared to the life situations related to their anxiety and other symptoms.

The most significant conclusion which arises from this and the earlier study of Wolf and Wolff (6) is that symptoms and signs of effort intolerance may occur in any person during periods of life stress and emotional disturbance. They may come and go repeatedly and their presence or absence at the time of examination will depend upon the life situation of the subject and also upon the circumstances and significance of the examination. Thus to certain patients the examination of their hearts may itself be stressful or be associated with decisions and implications which are stressful. Subjects who are capable of vigorous exertion may show gross intolerance to exercise during a stressful examination. An instance is the patient of Bishop and Kimbro (19) who on two occasions of army service 24 years apart showed tachycardia and gross intolerance to exercise, but who in the interval had engaged in the heavy physical exertions of running a successful farm (20). At the time of his army examinations he showed in ad- 
dition to circulatory inefficiency, evidences of great anxiety. Conversely the hospital or clinic may represent to some a friendly protective atmosphere in which the patient feels relaxed and at ease and therefore no explanation may be found from physical examination or tests of cardiac function for the troublesome symptoms of which the patient complains.

Such effort intolerance related to life stress may overlie or magnify effort intolerance related to structural heart diseases. It is particularly important in these conditions for several reasons. First it may be confused with the symptoms and signs of cardiac failure. Secondly it may evoke an unfavorable prognosis when not independently evaluated and thus may bring an intensification of anxiety and effort intolerance in the patient. Finally, the inefficient, rapid and excessive action of the heart may itself be detrimental to the course of the structural heart disease.

\section{SUMMARY AND CONCLUSIONS}

1. A study was made of heart rate, blood pressure, and cardiac output before and after a standard exercise test in subjects with and without structural heart disease who gave evidence of various degrees of emotional disturbance.

2. The average cardiac outputs of subjects who were slightly disturbed emotionally were greater before and after exercise than those of subjects who were apparently relaxed. The difference was largely attributable to increases in stroke volume.

3. The average cardiac outputs of subjects with marked, overt anxiety were greater before and after exercise than those of the poorly relaxed subjects. The difference was largely attributable to greater increases in heart rate.

4. In general there was a close correlation between symptoms such as dyspnea, palpitations and weakness on exertion and impaired exercise tolerance.

5. In ten subjects with structural heart disease studied similarly, the same relationship was found between emotional disturbances and the occurrence of symptoms and signs of effort intolerance. The symptoms themselves were similar to those associated with cardiac failure.

6. Changes in the emotional state were accompanied by changes in exercise tolerance and in seven instances such alterations in exercise tolerance were observed in less than an hour.

7. The following conclusions are drawn:

a) Impaired exercise tolerance during emotional disturbances apparently results from exaggerated cardiac mobilization in response to symbolic stimuli to action. In the early stages the increased cardiac output is mainly achieved by increases in stroke volume. In greater degrees, excessive increases in heart rate contribute more to the increase in cardiac output.

b) Ordinary physical exertions of everyday life performed during periods of relaxation and security impose little extra work on the heart compared to the cardiac mobilization associated with anxiety.

c) The increased cardiac work and excessive tachycardia at rest and in response to exercise during anxiety may be relevant to the increased susceptibility of patients with tachycardia to the development of structural heart disease.

\section{ACKNOWLEDGMENT}

The authors wish to thank Miss Dorothy Dingfeld and Mr. David Greenberg for valuable technical assistance in this study.

\section{BIBLIOGRAPHY}

1. Jones, M., and Mellersh, V., A comparison of the exercise response in anxiety states and normal controls. Psychosom. Med., 1946, 8, 180.

2. Rothschild, M. A., Neurocirculatory asthenia. Bull. N. Y. Acad. Med., 1930, 6, 223.

3. Meakins, J. C., and Gunson, E. B., The pulse rate after a simple exercise test in cases of "irritable heart." Heart, 1917, 6, 285.

4. Jones, M., and Scarisbrick, R., Effect of exercise on soldiers with neurocirculatory asthenia. Psychosom. Med., 1946, 8, 188.

5. Friedman, M., Studies concerning the etiology and pathogenesis of neurocirculatory asthenia. III. The cardiovascular manifestations of neurocirculatory asthenia. Am. Heart J., 1945, 30, 478.

6. Wolf, G. A., Jr., and Wolff, H. G., Studies on the nature of certain symptoms associated with cardiovascular disorders. Psychosom. Med., 1946, 8, 293.

7. Nickerson, J. L., and Curtis, H. J., The design of the ballistocardiograph. Am. J. Physiol., 1944, $142,1$.

8. Nickerson, J. L., Warren, J. V., and Brannon, E. S., The cardiac output in man: studies with the low 
frequency, critically-damped ballistocardiograph and the method of right atrial catheterization. J. Clin. Invest., 1947, 26, 1.

9. Master, A. M., and Oppenheimer, E. T., A simple exercise tolerance test for circulatory efficiency with standard tables for normal individuals. Am. J. Med. Sc., 1929, 177, 223.

10. Nickerson, J. L., Personal communication.

11. Stevenson, I., and Duncan, C. H., Variations in exercise tolerance with changes in the emotional state. To be published.

12. Cannon, W. B., Bodily Changes in Pain, Hunger, Fear, and Rage. D. Appleton and Co., N. Y., 1929, 2nd Edition.

13. Hickam, J. B., Cargill, W. H., and Golden, A., Cardiovascular reactions to emotional stimuli. Effect on the cardiac output, arteriovenous oxygen difference, arterial pressure, and peripheral resistance. J. Clin. Invest., 1948, 27, 290.

14. Levy, R. L., White, P. D., Stroud, W. D., and Hillman, C. C., Transient tachycardia. J. A. M. A., 1945, 129, 585.

15. Stevenson, I., and Duncan, C. H., Unpublished data.

16. Smith, H. W., Physiology of the renal circulation. Harvey Lectures, 1939-1940, 166.

17. Wolf, S., Pfeiffer, J. B., Ripley, H. S., Winter, O. S., and Wolff, H. G., Hypertension as a reaction pattern to stress. Summary of experimental data on variations in blood pressure and renal blood flow. Ann. Int. Med., 1948, 29, 1056.

18. Harrison, T. R., Failure of the Circulation. Williams \& Wilkins Company, Baltimore, 1935.

19. Bishop, L. F., and Kimbro, R. W., Neurocirculatory asthenia. J. A. M. A., 1943, 122, 88.

20. Bishop, L. F., Personal communication. 